\title{
AUTONOMOUS POSITIONING AND FULL TRAVERSAL PATH PLANNING TECHNOLOGY FOR INDOOR INTELLIGENT SWEEPING ROBOT
}

\author{
Yang Lin \\ Hebei Youth Management Cadre College, Hebei 050031, China \\ Email: yanglinjack1@126.com
}

\begin{abstract}
To reduce the complex cleaning workload, people developed indoor intelligent sweeping robot, which can help people clean the indoor environment intelligently and autonomously. Based on previous studies, the current conventional technologies of sweeping robot are introduced, and further studies are carried out. Firstly, low-cost photoelectric encoder and MPU6050 sensor combination positioning are selected for the robot to conduct modeling for the sensor, the system error and random error in the positioning process are analysed, and different correction methods are adopted. Secondly, the system error is corrected by analytical method, and the random error is corrected by extended Kalman filter algorithm. The feasibility of the algorithm is verified by simulation, which provides a guarantee for the research of full traversal path planning. For the area connection path planning of irregular obstacles between adjacent partitions, the sensor is used to clean the irregular obstacles along the side, so that the expansion area can also be covered for cleaning, and the overall cleaning coverage rate can be improved. In order to reduce the repetition rate of the regional connection path between regular obstacles, the improved $A^{*}$ algorithm is used to obtain the rules of starting point, direction and ending point of round-trip traversal in rectangular partition, and the round-trip artificial cleaning mode is used in the rectangular partition without obstacles. The combination of Manhattan distance and diagonal distance is introduced, and the corner cost is introduced into the evaluation function, which effectively reduces the repetition rate of regional connection path. Finally, RobotBASIC is used to carry out the simulation experiment. The results show that the proposed method enables the cleaning robot to find full coverage, low repetition rate and efficiently traverse the cleaning path. The results show that the proposed method enables the cleaning robot to find a traverse clean path with full coverage, low repetition rate and high efficiency.
\end{abstract}

Keywords: Sweeping Robot; Autonomous Positioning; Full Traversal Path Planning.

\section{Introduction}

In 1959, the first robot was born. In March 2016, Google's AI robot A1phaGo defeated the world go champion Lee Sedol, which witnessed the rapid development of robot technology. Robots are mainly used to improve working conditions of human, improve productivity, and solve or replace human beings to complete more difficult, more accurate and more onerous production problems [1]. After several stages of development of industrial robots, the first generation of robots consists of programmable controllers, servo systems, manipulators, etc., and the robots are programmed to achieve simple fixed operations; the second generation of robots is invented on the basis of the first generation of robots, plus infrared, ultrasonic, vision, pressure and other sensors, so that the robots have a certain degree of environmental awareness. According to the information perceived in real time, the robots can make corresponding adjustments. Robots that are currently used on a large scale in the factory meet these characteristics; the third generation of robots are more intelligent than the second generation [2]. With the addition of artificial intelligence algorithm, the robots have the ability to perceive and think at the same time. It can adapt to a more complex environment and complete complex tasks more intelligently [3]. Some of the service robots now belong to the third generation of robots [4].

With the development of science and technology, science fiction has gradually become a reality [5]. And with the rapid development of intelligent robots, the use of robots has expanded from a single factory to various fields [6]. On the basis of solving the production efficiency of factories, robots gradually replace people's work in unknown or unconventional environments. When a robot explores an unknown and unstructured 
environment, it requires a higher degree of intelligence. The development of computer technology and sensor technology provides more possibilities for such robots and also enables the practical use of intelligent robots to expand from the land to the seabed and then to space [7]. Based on the development of technology, service robots are developing according to people's requirements for the quality of life [8]. In addition to the above highend application fields which are far away from daily life, service robots have become a research hotspot in recent years [9].

For different application backgrounds, service robots can be divided into the following types: tourist display robot, interpretation robot; ordering and delivering robots for restaurant service; medical rehabilitation robot, escort robot and surgical robot; security robot, fire rescue robot; warehouse logistics robot; indoor intelligent cleaning robot, external wall intelligent cleaning robot, etc. Intelligent cleaning robot has the widest development prospect [10].

The pace of contemporary life is accelerating, the cost of time is becoming more expensive, and modern families that young people are the mainstay are even more reluctant to clean up indoor hygiene [11]. The birth of intelligent cleaning robot provides a new solution for indoor cleaning. It releases our hands and has the function of sweeping and mopping the floor, which can complete the boring and heavy cleaning work for us. Compared to home use, cleaning of offices, shopping malls, warehouses, etc., can also save a lot of labor costs due to smart cleaning robots [12]. The key and difficult point of intelligent cleaning robot is path planning [13]. How to make intelligent cleaning robot consume less energy, achieve full traversal cleaning in a shorter time, and minimize the repetition rate has been the key and difficult point of research [14]. The path planning of intelligent cleaning robots is universally representative and has a reference function for path planning of other types of robots. For example: pesticide spraying robot, fruit tree picking robot, oil tank cleaning robot and so on [15]. Therefore, it is necessary to study path planning for intelligent cleaning robots, which is conducive to promoting the application and popularization of intelligent cleaning robots and such mobile robots and has significant social effects and application prospects.

\section{Key Technology Analysis of Sweeping Robot}

The goal of indoor intelligent sweeping robot is to basically achieve intelligence and autonomy, that is, in the absence of human intervention, it can independently complete the indoor cleaning task. Autonomous positioning and full traversal path planning are the key technologies for sweeping robot. In this study, the existing positioning and full traversal path planning technologies are introduced and analyzed, the requirements of the sweeping robot are analysed, and the corresponding index requirements are specified, laying a foundation for the subsequent detailed study of key technologies.

\subsection{Common key technologies of sweeping robot}

Sweeping robot is composed of sensing detection system, control system, mobile and dust collection system. Its key technologies include sensor technology, positioning technology, full traversal path planning technology and autonomous recharging technology. In this study, the autonomous positioning and full traversal path planning technologies are mainly explored, so the existing achievements of these two key technologies are firstly introduced.

Sensor technology. The sensor is equivalent to the sense organs of the robot. When the robot is in operation, it needs to acquire its own posture and detect the external environment in real time, so as to to achieve the purpose of creating an environmental model, autonomous navigation, positioning, obstacle avoidance, path planning and checking cleaning effect. Sensors are usually divided into internal sensors and external sensors. Among them, the internal sensors include gyroscope, electronic compass, linear accelerometer, and encoder; and external sensors include laser sensors, infrared sensors, ultrasonic sensors, visual sensors, proximity and contact sensors. The development level of sensor technology greatly affects the intelligence level of robots. Due to the limitations of the function of a single sensor and its own resolution and other factors, comprehensive and reliable information can't be obtained, so it is often necessary to improve the accuracy of information through multi-sensor information fusion technology. Multi-sensor information fusion technology uses information complementation of various types of sensors to eliminate asymmetry and coupling information, and ultimately obtain reliable and effective information. Within the research scope of sensor technology, there are various multi-sensor information fusion technologies, such as Kalman filtering, Bayesian Estimation, D-S evidence theory and weighted average method.

Autonomous positioning technology. In the research of sweeping robot, autonomous positioning is a basic and important key technology, which is the premise of environmental map creation and path planning. It can be divided into relative positioning method and absolute positioning method. Absolute positioning method often adopts satellite navigation technology (GPS), passive or active identification, map matching or navigation beacons and other methods for positioning, and its positioning accuracy is high. 
Among these methods, GPS is mainly used for outdoor positioning, the construction and maintenance cost of signboard or beacon is high, and the processing speed of map matching technology is slow; position calculation methods of absolute positioning include three sight distance method, model matching method and three view method. The relative positioning, that is, the dead reckoning, estimates the current position of the robot by measuring the distance and direction of the robot relative to the initial position. Commonly used sensors include inertial navigation systems (accelerometers, gyroscopes, etc.) and encoders. The advantage of the dead reckoning is that the robot's posture is calculated by itself, and it doesn't need to perceive the external environmental information. The disadvantage is that the drift error will accumulate over time, which is not suitable for accurate positioning.

Path planning technology. The path planning of the sweeping robot is a basic and complex problem in robotics. It refers to that the robot, based on the perceived environmental information, plans a path between the starting point and the ending point that does not collide with the environmental space obstacles and traverses the whole region according to some optimization performance indexes. It refers to a path that is non-collision with the environmental space obstacles and can achieves full-area traversal planned by the robot according to the perceived environmental information and based on some optimization performance index between the starting point and the ending point. Therefore, full traversal path planning is the core and key technology of the sweeping robot. At present, the full traversal path planning method of the sweeping robot can be divided into the path planning method based on the unknown environment and the path planning method based on the known environment.

Path planning methods based on unknown environment mainly include random type, circuitous type along the edge and internal and external spiral type. For the space without obstacles, the traversal of the whole region can be realized, but if there are obstacles and the environment space is complex and changeable, these methods can't meet the requirements. At present, most of the coverage methods of sweeping robots in the market are random methods, such as Electoulux's Trilobita, Karcher's RC3000 and iRobot's Roomba.

Path planning methods based on known environments include template method, unit decomposition method, artificial potential field method and biological excitation method. The motion template method is to divide the possible environmental conditions into different templates and execute the corresponding templates when the corresponding conditions are encountered in the cleaning process. The unit decomposition method includes three processes: the division of sub-regions, the connection of sub-regions and the traversal of sub-regions. Firstly, according to the distribution of obstacles, the environmental regions are divided into a limited number of non-overlapping and barrierfree regions. Then, artificial intelligence methods such as genetic algorithm and neural network are used to connect the paths of sub-regions. The traversal of subregions often adopts template method, but it will cause repeated traversal due to the division of many regions. The artificial potential field method divides the environmental space into target area and obstacle area, which are described by gravitational field and repulsion field respectively.

The target area generates gravitation to the robot, and the obstacle area generates repulsion to the robot and traverses the entire environment space, but it often falls into local minimum points. Based on the raster map, the biological excitation method corresponds the discrete coordinates of twodimensional space with the neurons of the neural network. Each grid corresponds to a biological neuron. It provides different suppression and excitation for the input of obstacles and nonobstacles to neurons, calculates the output of relevant neurons, and then judges the next moving position of the robot to realize the whole region traversal.

\subsection{Analysis of existing key technologies}

At present, the key technology research of indoor intelligent sweeping robot has achieved good results, but there are still many problems to improve. In this study, two key technologies of autonomous positioning and full traversal path planning of sweeping robot are introduced, and the methods of positioning and full traversal path planning are proposed after contrastive analysis. At the same time, the requirements for the positioning and full traversal path planning of the sweeping robot are proposed, the requirements analysis is carried out, and the corresponding index requirements are put forward.

Selection of sensors: in order to complete the cleaning work of the floor autonomously, the sensor on the robot is indispensable, and it is a direct device to obtain its own posture and environmental information. At present, ultrasonic sensors, laser sensors and infrared sensors are used for ranging. And the electronic compass, gyroscope and MPU6050 are used to measure azimuth.

Sensor distribution design: the sensor distribution of the sweeping robot is shown in figure 1. The ultrasonic ranging sensor is used to detect the surrounding environment information. The ultrasonic ranging module is set in the left, middle and right directions of the robot. In order to avoid the mutual interference between the transmitting signal and the echo signal of each module, each ultrasonic module is started in the mode of 
alternating triggering; the infrared sensor is used to avoid obstacles, and evenly arrange 7 infrared sensors in the range of $180^{\circ}$ in front of the robot; collision switch plays a protective role in the case of failure of ultrasonic sensor and infrared sensor. The photoelectric encoder is installed on the two driving wheels to detect the walking distance and azimuth of the robot; at the same time, the angular velocity and acceleration measured by MPU6050 are integrated to obtain the angle and position for data fusion with the photoelectric encoder to achieve more accurate positioning.

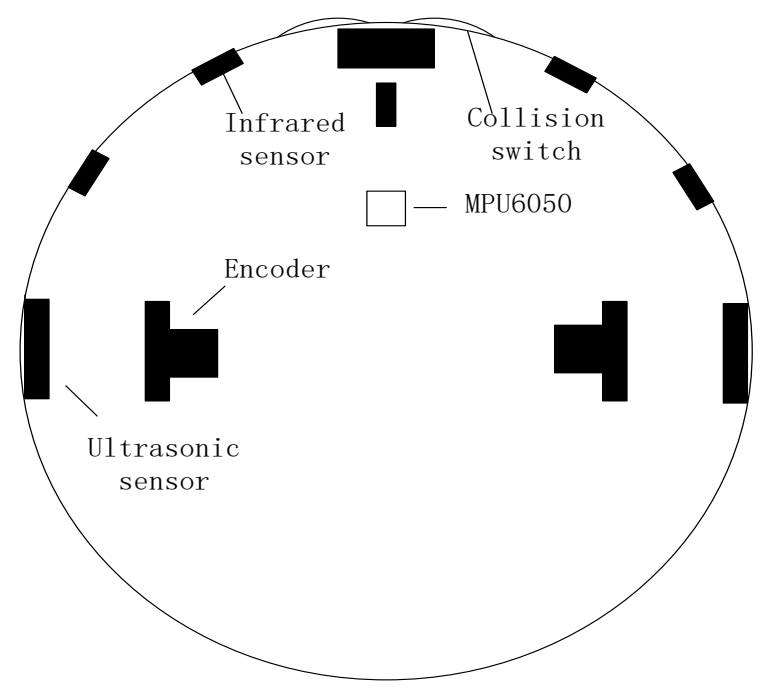

Figure 1. Sensor distribution diagram

Analysis of positioning method: positioning is a method in which a robot acquires information through a sensor and determines its own posture through data processing. At present, indoor positioning methods include map matching positioning, signpost identification positioning and dead reckoning positioning.

Map matching positioning is to determine the posture of the robot by comparing the local information acquired by the sensor with the information in the known environment map. However, prior map of the environment space needs to be stored in the robot in advance. In addition, map matching positioning often need to be combined with other positioning methods to achieve positioning. Due to the experimental conditions and the limitations of the robot's own hardware, this method is not applicable. Signage identification positioning are dependent on a series of sign ages with known features in the environment, which are equipped with detection devices on the robot and positioned by trilateration or triangulation. Ultrasonic sensors, visual sensors and laser sensors can be used as signposts. However, due to the high cost of installation and maintenance, and the difficulty in popularizing it to consumers' homes, these sensors are not applicable.
The dead reckoning method is a method widely used in robot positioning. The most commonly used sensor is the photoelectric encoder. Its advantage is that high localization accuracy can be provided without external information, and the disadvantage is that the error accumulates with time. In order to improve the positioning accuracy and stability of the dead reckoning method, various sensors are often used to reduce the influence of non-systematic errors on robot positioning by information fusion. In this study, this method is used to eliminate the random error of the sensor for positioning.

Analysis of full traversal path planning method: full traversal path planning is the core technology of sweeping robot. According to some path planning algorithm, the robot traverses the accessible area of the entire environment space and ensures the maximum coverage rate and the minimum repetition rate. At present, the most commonly used cleaning methods include random type, circuitous type along the edge and inner spiral. Random type is shown in Figure 2, the robot moves along a straight line, randomly rotates an angle to continue moving when encountering obstacles or boundaries.

This method has low requirements for sensors and controls, but it has high repetition rate and low efficiency. Circuitous type is shown in figure 3, robot moves in a certain direction. After encountering an obstacle or boundary, it turns 90 degrees and moves sideways for a distance, then turns 90 degrees to move. It is simple to control, and the traversal path is regular, but it is easy to enter a dead angle or cause some areas to be missed when there are many obstacles. Inner spiral type is shown in Figure 4, the robot moves along the edge, takes obstacle avoidance measures when encountering obstacles, and carries out "HUI" movement. It is mainly used for local area cleaning, but the turning point needs to be set.

These three cleaning methods are easy to control and are suitable for simple environment space, but the actual environment space is more complex.

To achieve better traversal effect, it is necessary to establish an environment model and combine path planning algorithm with common cleaning methods to achieve full traversal path planning.

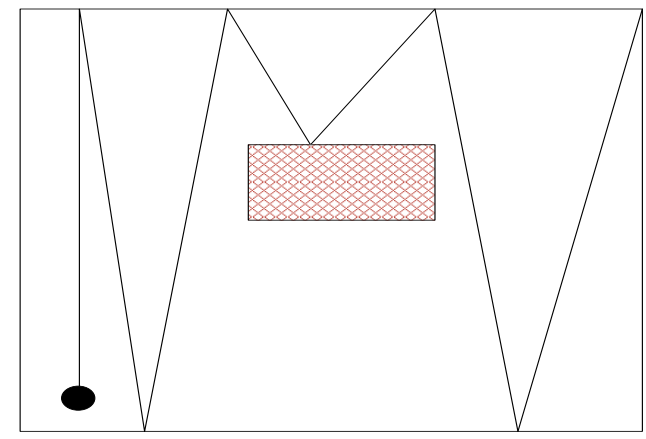

Figure 2. Random type 


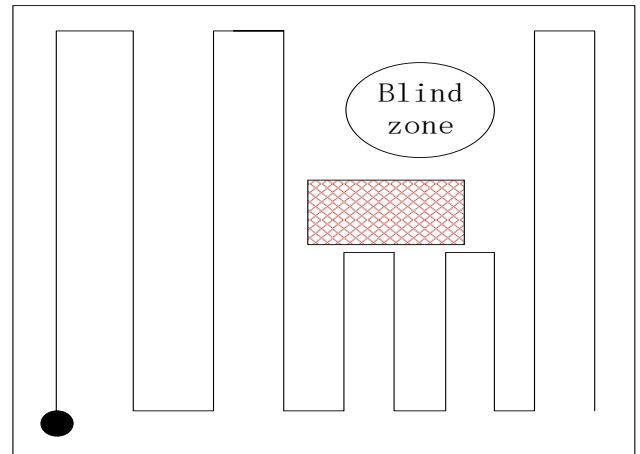

Figure 3. Circuitous type

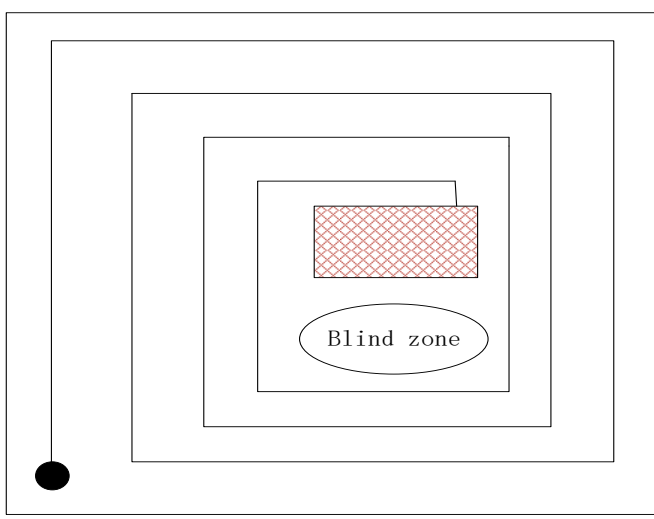

Figure 4. "HUI" type

Environment modeling mainly includes raster method, geometric feature method and topology method. For indoor sweeping robot, raster map is easy to create and maintain and is widely used. At present, common intelligent algorithms for full traversal path planning include artificial potential field method, fuzzy logic algorithm, neural network algorithm, etc.

The artificial potential field method traverses the whole space through the attraction of the target region to the robot and the repulsive force of obstacles to the robot, but it is easy to fall into the local minimum; fuzzy logic algorithm is combined with other methods to improve the planning effect by setting fuzzy rules. It is highly practical for lowcost robots, but the complex environment will lead to a sharp expansion of fuzzy rules; the biological excitation neural network algorithm proposed by S.X.Yang according to the shunt model is the most widely used neural network algorithm. It doesn't need to learn, has good real-time performance and high coverage rate, but it has problems such as large randomness, high repetition rate, low efficiency of dead angle escape and complex calculation. For the above analysis of the full traversal path planning method, there are mainly problems such as low coverage caused by blind spot missing sweeping, high repetition rate caused by repeated cleaning, and low escape efficiency after entering the dead angle.

Aiming at the above problems, an improved neuron excitation algorithm based on biological excitation neural network is proposed for the unknown static environment, which has the advantages of high coverage rate, low repetition rate, simple and fast operation.

\subsection{Requirement analysis and index requirement of sweeping robot}

Requirement analysis: indoor intelligent sweeping robot replaces human to complete tedious and repetitive floor cleaning work, which is more and more popular among people. The sweeping robot shall meet the following requirements: strong practicality, self-contained large-capacity battery, simple operation, light and compact, and strong autonomy; the working environment is mainly the indoor environment of ordinary families, which requires strong environmental adaptability; positioning in the environment is accurate, and it can complete the full traversal cleaning of the environment as much as possible. Meanwhile, it can safely and reliably avoid obstacles to prevent damage to the home and have the ability of selfprotection.

Standard requirement: the positioning and full traversal path planning tasks completed by the sweeping robot are important indexes to evaluate the product performance of the sweeping robot.

The existing sweeping robots in the market are analyzed and the following indicators are set: related sensor error and positioning error: the ranging error of the ultrasonic sensor is within \pm 1 ; the positional error of autonomous positioning is $\pm 5 \mathrm{~cm}$ and the azimuth error is within $\pm 3^{\circ}$. The sweeping robot is fully traversed: coverage rate is greater than or equal to $95 \%$, repetition rate is less than or equal to $10 \%$; reasonable walking strategy is adopted to minimize energy consumption.

The robot's rescue time is less than or equal to 1 $\min$, the success rate of rescue is more than or equal to $90 \%$, and the anti-fall error rate is less than or equal to $10 \%$. Through performance optimization and algorithm improvement, the cost can be reduced, and the function of the sweeping robot can be realized.

\section{Research on the Autonomous Positioning of Indoor Intelligent Sweeping Robot}

To be able to accurately locate itself in the working environment is the basis for the sweeping robot to establish a map of the unknown environment and the premise of realizing the full traversal path planning. Positioning can determine the position and pose of the robot relative to the global coordinates in the two-dimensional workspace, which is the most basic condition for its navigation. In this section, the autonomous positioning technology of sweeping robot is studied in detail and the systematic error 
and random error in the positioning process are corrected and analyzed.

\subsection{Positioning sensor model and system error correction}

The robot positioning system is realized by a photoelectric encoder mounted on two driving wheels and an internal MPU6050 six-axis sensor.

Photoelectric encoder is a kind of sensor that converts the mechanical geometric displacement on the output shaft into digital quantity or pulse through photoelectric conversion, including incremental encoder and absolute encoder.

The absolute encoder has memory function after power off and can record the position of the encoder in an absolute coordinate system, but the cost is high; incremental encoders can output incremental changes from a predefined starting position. In order to use the output signal to determine the direction of motion, it usually chooses the double channel incremental encoder, which is easy to integrate and maintain, cheap and widely used. Double channel incremental encoder mainly includes shaft, main dial, luminous element, photosensitive element and conversion circuit. The incremental encoder outputs pulses proportional to the angular increment.

The number of pulses is measured by a timer to obtain the distance traveled by the left and right wheels, and it has no fixed starting point. The output of incremental encoder is proportional to the pulse of the angular increment. The number of pulses is counted by the timer to obtain the distance traveled by the left and right wheels, and it has no fixed starting zero.

Taking the left driving wheel as an example, suppose that the diameter of the wheel is $D_{l}$, the number of lines of the photoelectric code plate is $n$, and the number of recorded pulses in time $\Delta \mathrm{t}$ is $\mathrm{N}_{1}$, then the driving distance of the driving wheel in time $\Delta \mathrm{t}$ is $\Delta \mathrm{S}_{l}$.

$$
\Delta \mathrm{S}_{l}=\frac{\pi \mathrm{D}_{l} N_{l}}{n}
$$

Let the moving distance of left and right wheels in time $\Delta \mathrm{t}$ be $\Delta \mathrm{S}_{l}$ and $\Delta \mathrm{S}_{\mathrm{r}}$ respectively, and the wheelbase of the two wheels be $B$, then the robot's distance $\Delta \mathrm{S}$ and the rotation angle $\Delta \theta$ are shown in the following equation:

$$
\left\{\begin{array}{l}
\Delta \mathrm{S}=\frac{\Delta \mathrm{S}_{l}+\Delta \mathrm{S}_{\mathrm{r}}}{2} \\
\Delta \theta=\frac{\Delta \mathrm{S}_{r}-\Delta \mathrm{S}_{\mathrm{r}}}{B}
\end{array}\right.
$$

Let the pose of the robot at time $k-1$ be:

$$
\left[\begin{array}{lll}
x(k-1) & \mathrm{y}(\mathrm{k}-1) & \theta(\mathrm{k}-1)
\end{array}\right]^{T}
$$

The pose at time $\mathrm{k}$ can be obtained with the dead reckoning method, as shown in the following equation:

$$
\left\{\begin{array}{l}
x(k)=x(k-1)+\frac{\Delta S(k-1)}{\Delta \theta(k-1)} \\
(\sin (\theta(k-1)+\Delta \theta(k-1))-\sin (\theta(k-1))) \\
y(k)=y(k-1)+\frac{\Delta S(k-1)}{\Delta \theta(k-1)} \\
(\cos (\theta(k-1)+\cos (\theta(k-1)+\Delta \theta(k-1))) \\
\theta(k)=\theta(k-1)+\Delta \theta(k-1)
\end{array}\right.
$$

When the robot moves in a straight line, $\Delta \theta(k-1) \rightarrow 0$, then the pose is:

$$
\left\{\begin{array}{l}
x(k)=x(k-1)+\Delta \mathrm{S}(k-1) \cos (\theta(k-1)) \\
\mathrm{y}(k)=y(k-1)+\Delta \mathrm{S}(k-1) \sin (\theta(k-1)) \\
\theta(k)=\theta(k-1)
\end{array}\right.
$$

The single sensor of MPU6050 sensor model can't obtain comprehensive and reliable information due to its limited functions and its own resolution and other factors. In order to achieve more accurate positioning of the robot, the MPU6050 six-axis sensor with low price and good performance is adopted as a complementary sensor.

As shown in Figure 5, the direct output value is the acceleration and angular velocity in three directions. Since the robot moves in a twodimensional plane, it only needs to read out the angular velocity of the Z-axis and the acceleration of the X-axis and Y-axis through cyclic sampling.

Then, the azimuth of the robot can be obtained by integrating the angular velocity of the Z-axis once, as shown in equation 6; the quadratic integration of the acceleration of the X-axis and Y-axis can obtain the position of the robot, as shown in equation 7 .

The smaller the interval between integrations, the more accurate the result will be after the integration, but it will increase the calculation amount and the system memory consumption, so the appropriate integration time should be selected.

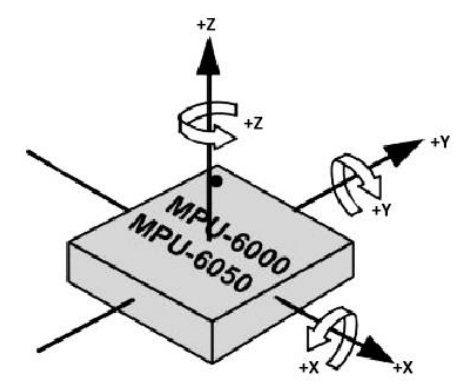

Figure 5. The principle diagram of MPU6050 
$\theta_{\mathrm{n}}=\theta_{n-1}+\omega_{n} d t$

Among them, $\theta_{\mathrm{n}}$ is the angle value at time $\mathrm{n}, \theta_{\mathrm{n}-1}$ is the angle value at time $n-1, \omega_{n}$ is the $\mathrm{Z}$-axis angular velocity read at time $\mathrm{n}$, and $\mathrm{dt}$ is the integration time.

$\left\{\begin{array}{l}v_{n x}=v_{(n-1) x}+a_{n x} d t \\ s_{n x}=s_{(n-1) x}+v_{n x} d t\end{array}\right.$

Among them, $\mathrm{v}_{n x}$ and $\mathrm{s}_{n x}$ are the velocity and displacement of the $\mathrm{X}$-axis at time $\mathrm{n}, \mathrm{a}_{n x}$ is the acceleration of $\mathrm{X}$-axis at time $\mathrm{n}, \mathrm{dt}$ is the integration time, and the velocity and displacement of the Y-axis at time $\mathrm{n}$ are similar to the above formula.

Angular velocity error correction: angular velocity error correction MPU6050 is affected by the manufacturing process and environment of inertial devices, so its calibration factor, zero offset and installation errors seriously affect its accuracy.

By analyzing and correcting errors, the measurement accuracy of the system can be effectively improved and the azimuth of the robot can be accurately measured in real time. Since the output angular velocity of MPU6050 is not zero at rest, there is a zero offset. If no effective method is adopted, the error will be amplified by accumulation. The zero-offset data is often read at rest with the mean filtering method and then compensated by a mathematical model, as shown in equation 8:

$\theta_{\mathrm{n}}=\theta_{n-1}+\left(\omega_{n}-\omega_{0}\right) d t$

Among them, $\omega_{0}$ is the zero drift value of Z-axis angular velocity obtained by mean filtering at rest.

Meanwhile, due to installation error, calibration factor and other factors, coefficient correction method is adopted for calibration, as shown in equation 9. In order to prevent factors such as jitter and external interference, $-1^{\circ} / s<\omega<1^{\circ} / s$ is discarded.

$\theta_{\mathrm{n}}=\theta_{n-1}+\mathrm{k}\left(\omega_{n}-\omega_{0}\right) d t$

Among them, $\mathrm{k}$ is the correction coefficient, which can be calculated by rotating the robot in situ several times.

Acceleration error correction: acceleration error correction is similar to the error of angular velocity output signal. The acceleration of $\mathrm{X}$-axis and Y-axis read by MPU6050 have static drift and environmental noise interference. Static drift is also eliminated by means of mean filtering.

Zero drift data of acceleration of X-axis and Y-axis are read in the static state of the robot, and then compensated by mathematical model, as shown in equation 10:

$\left\{\begin{array}{l}v_{n x}=v_{(n-1)}+\left(a_{n x}-a_{o x}\right) d t \\ s_{n x}=s_{(n-1) x}+v_{n x} d t\end{array}\right.$
Among them, $a_{o x}$ is the zero-drift value of the Xaxis acceleration obtained by mean filtering at rest, and the velocity and displacement of $\mathrm{Y}$-axis at time $\mathrm{n}$ are similar to the above formula [12]. Acceleration requires quadratic integral to obtain displacement, and the integral is equivalent to the product of the sampling value and the sampling time. Only when the sampling time $d t \rightarrow 0$, the data after the integration is approximately accurate.

The constant quadratic integration of acceleration makes the cumulative error larger, so the sampling loss needs to be reduced by compensation. As shown in Figure 6, the acceleration sampling loss is taken as an example for error compensation. Assume that the acceleration of the Xaxis at time $\mathrm{n}-1$ is $a_{(n-1) x}$, then the acceleration at time $\mathrm{n}$ is $a_{\mathrm{n} x}$, as shown in Equation 11.

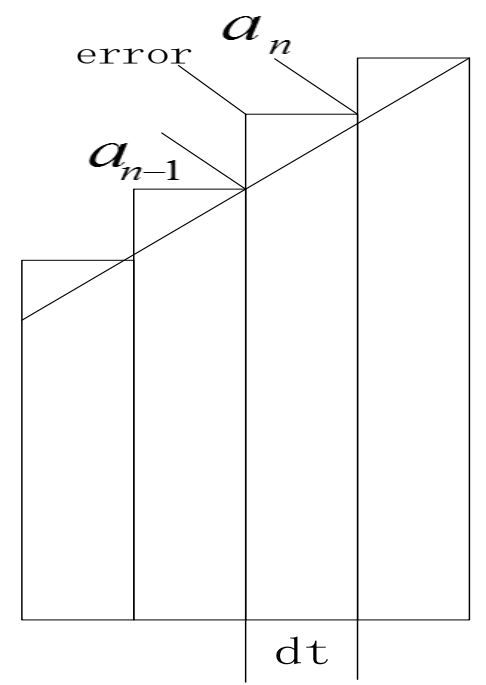

Figure 6. Method of sampling error compensation

$v_{n x}=v_{(n-1) x}+a_{n x} d t-\left|a_{n x}-a_{(n-1) \times}\right| d t / 2$

The zero drift of acceleration is eliminated by means of mean filtering, and the sampling compensation method is adopted to improve the sampling accuracy. The displacement obtained after the quadratic integration of acceleration is more accurate. Then the motion state detection method is used to determine: if the robot is at rest, the displacement remains unchanged and the velocity and acceleration are set to zero; if the robot is in the state of motion, the integral operation is carried out continuously, which can eliminate the fluctuation of acceleration signal. The robot pose data obtained by the MPU6050 is combined with the photoelectric encoder data to achieve more accurate positioning of the robot. 


\subsection{Multi-sensor fusion positioning}

In order to improve the accuracy of dead reckoning positioning and reduce the impact of random error of a single sensor, Kalman filter algorithm is used to fuse the photoelectric encoder and MPU6050 for positioning.

Kalman filter principle: Kalman filter is an algorithm that uses linear system state equation to estimate the system state optimally through prediction and correction. It is widely used in data fusion, navigation, computer image processing and missile tracking.

The prediction process refers to the prior estimation of the current state based on the optimal estimation of the previous moment and the established time update equation, and the calculation of the current state value and its error covariance; the process of correction is to obtain the optimal estimation of the current state through the state update equation based on the observed value and the previously obtained predicted value of the current state. Kalman filter is suitable for linear, discrete or finite dimensional systems, and extended Kalman filter is generally used for nonlinear systems.

Extended Kalman filtering principle: the extended Kalman filtering is to linearize the nonlinear process, and then implement with the Kalman filtering algorithm. Extended Kalman filtering is a recursive process that can perform an optimal estimation of state variables at any time.

Positioning process: the photoelectric encoder and MPU6050 sensor are used to locate the sweeping robot. If the ground is not level or the driving wheel is slipping, the encoder will generate random errors, while the MPU6050 will also generate drift errors. Although the error of sensor system is analyzed and calibrated, the influence of random error will also lead to inaccurate positioning or positioning failure.

Therefore, the extended Kalman filter is used to achieve the optimal estimation of location and reduce the random error.

The predicted value of robot pose is estimated based on the output information of the photoelectric encoder. Meanwhile, the observed value of robot pose at the current moment is obtained by MPU6050.

Then, the predicted value of robot pose is updated and corrected according to the observed value to obtain the optimal estimation of pose.

The detailed process is shown in Figure 7.

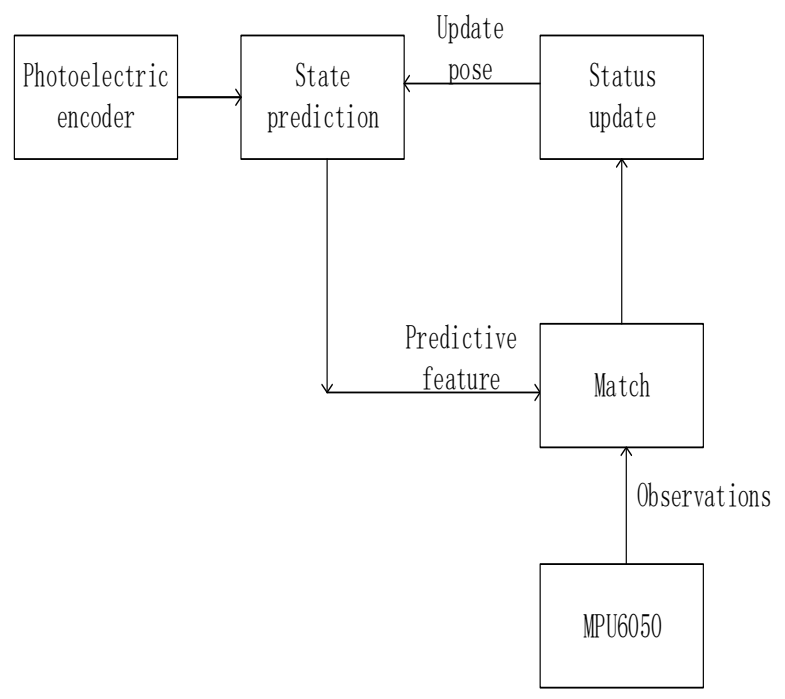

Figure 7. Positioning flow chart

Pose prediction: the optimal estimated pose of the encoder at the last moment is $\hat{X}(k-1)$, the input at this moment is $\mathrm{U}(k-1)$, then the estimated pose at the next moment is obtained, as shown in equation 12 :

$$
\begin{aligned}
& \hat{X}(k \mid k-1)=\mathrm{F}(\hat{\mathrm{X}}(k-1), U(k-1)+W(k-1) \\
& =\left[\begin{array}{l}
\hat{X}(\mathrm{k}-1)+\Delta \mathrm{S}(\mathrm{k}-1) \cos \hat{\theta}(\mathrm{k}-1) \\
\hat{\mathrm{y}}(k-1)+\Delta \mathrm{S}(\mathrm{k}-1) \sin \hat{\theta}(\mathrm{k}-1) \\
\hat{\theta}(\mathrm{k}-1)
\end{array}\right]
\end{aligned}
$$

Among them, $\mathrm{W}(\mathrm{k}-1)$ is Gaussian white noise with system noise, covariance of $Q$ and mean value of zero. The covariance is shown in Equation 13:

$$
p(k \mid k-1)=\nabla F P(k-1) \nabla F^{T}+q(k-1)
$$

Among them, $\nabla \mathrm{F}$ is the Jacobian matrix of the state model, as shown in equation 14 :

$$
\nabla F=\frac{\partial F(\hat{X}(k-1), U(k-1))}{\partial \hat{X}(k-1)}
$$

Prediction of observed quantities: MPU6050 model is an observation model, and the observation equation is shown in equation 15 :

$$
Z(\mathrm{k})=\mathrm{H}(\mathrm{X}(\mathrm{k}))+V(\mathrm{k})
$$$$
\text { Among them, } V(k) \text { is Gaussian white noise with }
$$
system observation noise, covariance of $R(k)$ and mean value of zero. Since the photoelectric encoder and MPU6050 are two sensors that independently measure the pose, the predicted observation value is the same as formula 12 , as shown in equation 16 :

$$
\hat{Z}(k \mid k-1)=H(\hat{X}(k \mid \mathrm{k}-1))+\mathrm{V}(\mathrm{k}-1)
$$


Among them, $\Delta H$ is the Jacobian matrix of the observation model, which is similar to formula 14; and the actual observation value $\mathrm{Z}(\mathrm{k})$ is obtained in real time by MPU6050.

Update: the Kalman gain, the pose optimal estimated value and the update covariance are calculated by pose prediction $\hat{X}(k \mid \mathrm{k}-1)$, actual observed value $Z(\mathrm{k})$ and observation predicted value $\hat{\mathrm{Z}}(k \mid \mathrm{k}-1)$.

The Kalman gain is shown in equation 17: $K(k)=$

$$
\begin{aligned}
& P(k \mid k-1) \nabla H^{T}\left(\nabla H P(k \mid k-1) \nabla H^{T}\right. \\
& +R(k-1))^{-1}
\end{aligned}
$$

Then, the optimal posture estimation and error covariance of the sweeping robot are shown in equations 18 and 19:

$$
\begin{aligned}
& \hat{X}(\mathrm{k})= \\
& \hat{X}(k \mid k-1)+\mathrm{K}(\mathrm{k}) \mathrm{Z}(\mathrm{k})-\hat{\mathrm{Z}}(\mathrm{k} \mid \mathrm{k}-1)) \\
& P(k)=(I-K(k) \nabla H) P(k \mid k-1)
\end{aligned}
$$

Extended Kalman filtering algorithm is to predict the current optimal estimation according to the state estimator and observation value at the previous moment, so as long as the estimation value at the initial moment is known, the optimal estimation of pose at any time can be obtained.

In this section, the autonomous positioning of the sweeping robot is mainly studied. Kinematics modeling of the sweeping robot is carried out with the three-wheeled model, and the photoelectric encoder and MPU6050 are used as the robot positioning sensor. The system error of the sensor is corrected by different methods, and the random error is reduced by data fusion with extended Kalman filter algorithm, so as to achieve more accurate positioning of indoor sweeper robot.

\section{Research on Full Traversal Path Planning Technology of Indoor Intelligent Sweeping Robot}

\subsection{Traversal path planning in a rectangular partition based on round-trip}

In Section 2, the planning method of traversal path is briefly introduced. The traversal path planning in the region mainly relies on the sensor's "return type", "round-trip" and template methods. Among them, "return type" and "round-trip type" only rely on the sensor's judgment of the front environment in free space to carry out corresponding motion control; the template method needs to match the stored template according to the sensor's judgment on the environment and traverse the cleaning with the corresponding template.
"Return type" and " round-trip" can also be used as cleaning templates, but the application scope of "return type" is limited, suitable for larger free area, not suitable for small space, so "return type" is the most commonly used.

When selecting the traversal method or traversing the template for path planning, it is first necessary to analyze the energy consumption of the smart cleaning robot under different motion modes.

The energy consumption of the motion during the cleaning process of the intelligent cleaning robot can be expressed as:

$$
E=E_{s}+E_{t}
$$

Among them, E represents the total energy consumption of the intelligent cleaning robot, $E_{s}$ represents the energy consumed by the movement in the straight direction, and $E_{t}$ represents the energy consumed during the turning movement of the intelligent cleaning robot.

When the intelligent cleaning robot performs linear motion, the driving force required for each unit length of travel is $F_{s}$. If the distance traveled in a straight line is $\mathrm{S}$, the energy $E_{s}$ consumed by the smart cleaning robot in straight travel can be expressed as:

$$
E_{s}=\int_{s} F_{s} d s=F_{s} S
$$

When the intelligent cleaning robot performs traversal cleaning such as "return type" and "roundtrip", the angle of each turn is basically the same.

Therefore, the energy consumed in each turning movement is the same, and it is set as $E_{o}$, then the number of traversal cleaning turns in an idle area is $\mathrm{N}$, so the energy $E_{\mathrm{t}}$ consumed by the intelligent cleaning robot in the turning movement can be expressed as:

$E_{t}=N E_{o}$

Therefore, the total energy consumption $\mathrm{E}$ of the intelligent cleaning robot during its travel is:

$$
E=F_{s} S+N E_{o}
$$

During the cleaning process of the path traversing by the intelligent cleaning robot, when the driving force $F_{s}$ required per unit length and the energy $E_{o}$ required for each turning are constant, the total energy consumed on the path of the intelligent cleaning robot is mainly determined by the total driving length and turning times. After modeling the working environment of the intelligent cleaning robot with raster method map, there is a certain number of grids in each rectangular partition. The robot needs to traverse and clean the free space after the partition and cover each free grid in the rectangular partition. Therefore, when the 
rectangular partition of raster map is finished, the robot in each rectangular partition traverses a constant path length. A complete turn process includes deceleration, turn and acceleration, and the process takes longer than straight time. Therefore, the number of turns determines the traversal cleaning time and energy consumption of the intelligent cleaning robot in the rectangular area. It should reduce the number of turns and save cleaning time and energy consumption.

As shown in figure 8 , the traversal paths of "round-trip" traversal methods in different directions in the same size area are compared.

Among them, the traversing environment is the rectangular free raster area of $5 \times 1$ grid, where the red traversal path indicates that the robot carries out "round-trip" traversal and cleaning along the long side of the rectangular partition, and the number of turns is 8; and the blue traversal path refers to the "round-trip" traversal and cleaning of the robot along the short side of the rectangular partition, and the number of turns is 18 . The relationship between the the number of turns $\mathrm{N}$ and the length and width of the rectangle is as follows:

With one grid as the unit length and $L \times W$ grids as the rectangular partition $(L \geq W)$, the intelligent cleaning robot carries out "round-trip" traversal along the long side $\mathrm{L}$, then the number of turns $N_{L}$ is:

$$
N_{L}=2 \times(\mathrm{W}-1)
$$

When the "round-trip" traversal is performed along the short side $\mathrm{W}$, the number of turns $N_{W}$ is:

$N_{W}=2 \times(L-1)$

Among them, $(L \geq W)$, so $N_{L} \leq N_{W}$. That is, the number of turns traversed by "round-trip" along the long side is less than the number of turns traversed along the short side.

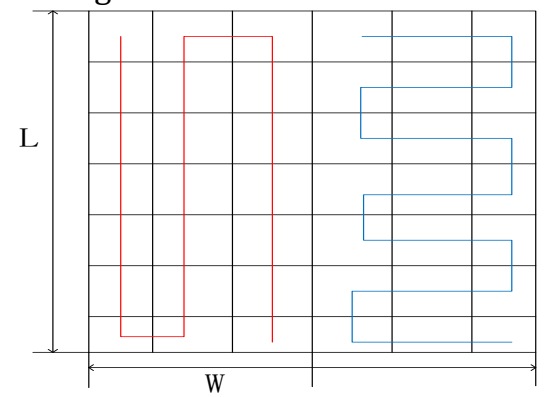

Figure. 8 The comparison of traversal paths in different directions for round-trip type

Determination of the start point and end point of traversal within the rectangular partition. For the planning of traversal path in rectangular partition, an intelligent cleaning robot traversal method of "round-trip" movement along the long rectangular edge is adopted.
This method not only imitates the manual sweeping method, but also facilitates the determination of the starting and ending points of the intelligent cleaning robot in the rectangular partition.

$\left(x_{1}, \mathrm{y}_{1}, x_{2}, \mathrm{y}_{2}\right)$ is used to represent each rectangular partition. The characteristics of the "round-trip" traversal method determine that the starting point and end point are at the four corners of the rectangular partition, as shown in the following equation:

$$
\left(\mathrm{x}_{1}, \mathrm{y}_{1}\right),\left(\mathrm{x}_{2}, \mathrm{y}_{1}\right),\left(\mathrm{x}_{2}, \mathrm{y}_{2}\right),\left(\mathrm{x}_{1}, \mathrm{y}_{2}\right)
$$

The starting point of traversal within the rectangular partition depends on the traversal order of the partition.

\subsection{Regional connection path planning}

After the rectangular partition, the intelligent cleaning robot carries out the traversal and cleaning for single rectangular partition.

According to the sequence of traversal of all partitions, it is necessary to move from a rectangular partition that has just been cleaned to the next rectangular partition that needs to be traversed and cleaned.

In the path planning between regions, obstacles are processed by expansion algorithm between regions, and edge traversal of these irregular edge areas is carried out with sensors of the intelligent cleaning robot. In order to reduce the repetition rate of the traversal path and improve the overall efficiency, the shortest path search algorithm is adopted.

The end point of the traversal of the above rectangular partition is taken as the starting point, and the starting point of the traversal of the next rectangular partition is taken as the target point for the search of the shortest path.

The irregular obstacle area connects the path planning, there is no obstacle in the partition, and the round-trip traversal cleaning mode is adopted.

The planning of connecting paths of irregular obstacle areas: if there is no obstacle in the partition, the round-trip traversal cleaning mode is adopted.

In order to achieve full coverage traversal cleaning of the overall environment, the edges of the irregular obstacles after the expansion process should be cleaned during the area connection.

For such irregular obstacles, in the planning of the partition connection path, it is necessary to use sensors to detect the edges of the irregular obstacles, control the distance between the robot and the obstacles, and clean along the edges of the irregular obstacles.

In the Robot BASIC simulation experiment, the toolbox has its own buffer, which can simulate ultrasonic sensors, infrared sensors, collision sensors, and so on. 


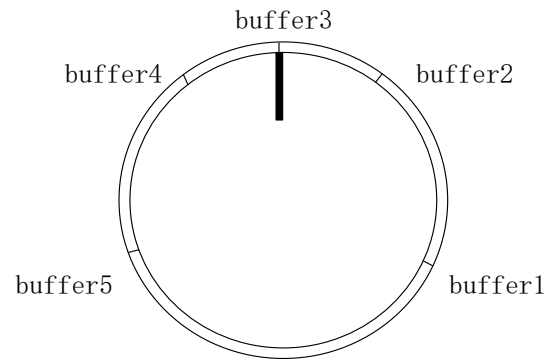

Figure 9.5 buffer positions in the Robot BASIC robot

In the Robot BASIC simulation environment, the positions of the five buffers that can be set as sensors in the robot are shown in figure 9. Buffer can read the distance between obstacles and set the safe collision distance, which not only protects the robot, but also can clean up to the edge of irregular graph.

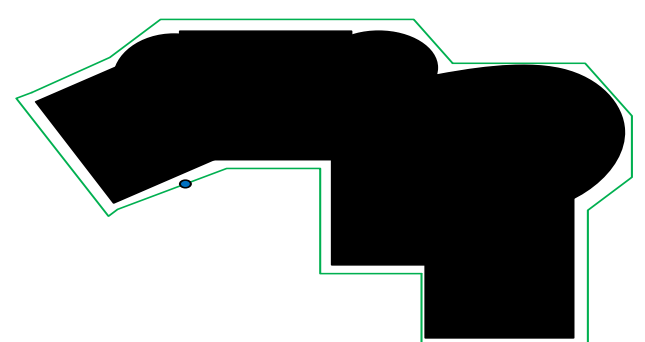

Figure 10. Simulation diagram of traversing path along irregular obstacles

As shown in Figure 10, RobotBASIC simulation environment simulates an irregular obstacle, that is, black irregular graph. Among them, the blue circle represents the robot, the green line represents the robot's detection of irregular obstacles by sensors, and then it traverses the robot's motion path around the edge of the irregular obstacles. Figure 10 shows that the robot can traverse along the edge of irregular obstacles by sensors.

The starting point and ending point in the rectangular partition are determined, and the improved $A^{*}$ algorithm is used to search the shortest path from the end point of the last traversed rectangular partition to the starting point of the next partition in the raster map. The path planning for the full traversal of the environment case is shown in figure 11.

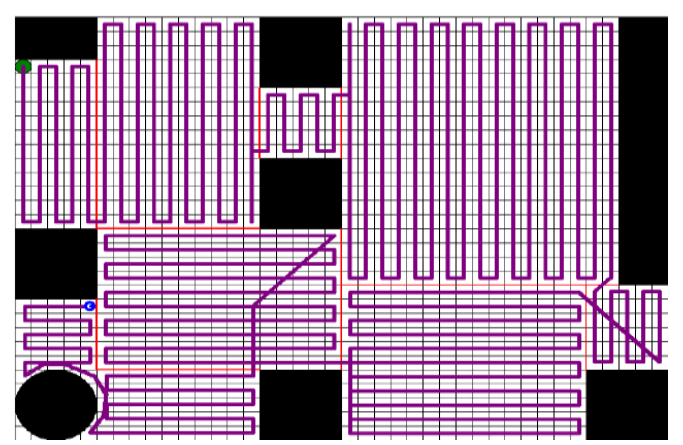

Figure 11. Full traversal cleaning trajectory of intelligent robot
Figure 11 shows the full traversal cleaning trajectory of the intelligent cleaning robot in the working environment. Among them, the diameter of the robot is the size of cell grid, and the green point is the starting point. The starting point, traversal direction and ending point of traversal path planning in rectangular partition are determined, "Roundtrip" path planning is adopted to traverse and clean each rectangular partition, and the improved $A^{*}$ algorithm is used to search for the shortest path between regions (from the ending point of the last rectangular partition to the starting point of the next partition), and finally the entire raster map is traversed and cleaned.

As shown in Figure 11, it consists of $40 \times 30$ grids, including 222 grids with removed obstacles, 998 free grids, and 49 grids with repeated traversals. The coverage is $100 \%$ and the repetition rate is $5 \%$.

Compared with the traditional rectangular partitioning algorithm, the improved rectangular partitioning algorithm has higher coverage and lower repetition rate. It improves the overall efficiency of rectangle partitioning algorithm. In this section, the "round-trip" traversal method in rectangular partition is introduced and analyzed, and the starting point, traversal direction and end point of traversal in the region are determined according to the "round-trip" traversal method in the partition. Aiming at the situation that the obstacles between regional junctions are irregular obstacles, sensors are used to clean the edges of such irregular edges treated with expansion. The obstacles in the regional connection are regular obstacles. The $A^{*}$ algorithm is used to search the shortest path between the regional connection, and the evaluation function is improved to obtain an improved $A^{*}$ algorithm more suitable for raster maps. In addition, simulation is carried out to verify that the improved $A^{*}$ algorithm can search out the path between regions with lower cost and reduce the repetition rate between regions.

\section{Conclusion}

With the development of science and technology and the improvement of people's living standard, the application of domestic service robot, especially indoor intelligent sweeping robot, is becoming more and more extensive. And the research of its key technology has attracted the attention of many scholars. As two core technologies in the research of sweeping robot, autonomous positioning and full traversal path planning have become the basis for realizing its full autonomy and intelligence. On the basis of fully understanding and analyzing the research status quo and common key technologies of sweeping robot at home and abroad, the positioning and full traversal path planning of indoor sweeping robot are deeply discussed and studied. 
In this study, the low cost photoelectric encoder and MPU6050 sensor are used to realize the precise positioning of the sweeping robot in the environment. The causes of sensor system errors are analyzed and concrete measures are taken to correct them. The random error is reduced by data fusion with extended kalman filter algorithm, and the feasibility of the algorithm is verified by simulation.

Aiming at the characteristics of no obstacles in the rectangular partition, the "round-trip" artificial traversal method is used to traverse and clean the rectangular partition. This type of movement is simple and can effectively reduce the cleaning time.

According to the edge length property of rectangle and the traversal order of partition, the starting point, direction and ending point of traversal within partition are determined, so as to make preparations for the path planning of crossregion.

The obstacles in the connecting area of rectangular partition are irregular obstacles. Depending on the sensor, the obstacle-free method is used to clean irregular obstacles and improve coverage rate. The inter-partition is the area connection of the regular obstacles. In order to reduce the path repetition rate of the cross-interval robot, the combination of Manhattan distance and diagonal distance is introduced, and the improved $A^{*}$ algorithm is used to improve the path planning efficiency across the interval. Finally, the intelligent sweeping robot is fully traversed in the overall working environment of multiple obstacles.

The result is better than the traditional traversal algorithm, achieving $100 \%$ full coverage, which effectively reduces the repetition rate of traversal and improves the overall cleaning efficiency of intelligent sweeping robot.

\section{Acknowledgement}

Research startup subject of Yangtze Normal Universi ty: 2017KYQD16

\section{References}

[1] Schwienbacher C, Meiners F, Kötter, Tobias, et al. Improvement of Multi-Directional Positioning Path Accuracy of Industrial Robots with Rotary Tables. Applied Mechanics and Materials, 2016, 826:6.

[2] Zou Y, Xu Y, Hu L, et al. Minimally invasive positioning robot system of femoral neck hollow screw implants based on x-ray error correction. Proceedings of the Spie, 2017, 245:102450D.

[3] Zahid S, Hobson P R, Cockerill D J A. A segmented anode vacuum phototriode with position sensitivity. Journal of Instrumentation, 2018, 13(01):C01014-C01014.
[4] Reiner B, Bownes P, Buckley D L, et al. Quantifying the trigger level of the vacuum surveillance system of the Gamma-Knife eXtend ${ }^{\mathrm{Tm}}$ positioning system and evaluating the potential impact on dose delivery. Journal of Radiosurgery \& Sbrt, 2016, 4(1):31-42.

[5] Lehman C D, Shook J E. Position of clip placement after vacuum-assisted breast biopsy: is a unilateral two-view postbiopsy mammogram necessary? Breast Journal, 2015, 9(4):272-276.

[6] Ayhan Üngördü, Tezer N. Effect on frontier molecular orbitals of substituents in 5-position of uracil base pairs in vacuum and water. Journal of Theoretical and Computational Chemistry, 2017, 16(07):7.

[7] Ashwal E, Wertheimer A, Aviram A, et al. The association between fetal head position prior to vacuum extraction and pregnancy outcome. Archives of Gynecology and Obstetrics, 2016, 293(3):567-573.

[8] Janicki, Ramírez-López, Manolo, Misiewicz J , et al. Sensitivity of Fermi level position at Ga-polar, Npolar, and nonpolar $\backslash r, m \backslash r$, -plane GaN surfaces to vacuum and air ambient. Japanese Journal of Applied Physics, 2016, 55(5S):05FA08.

[9] Schwienbacher C, Meiners F, Kötter,Tobias, et al. Improvement of Multi-Directional Positioning Path Accuracy of Industrial Robots with Rotary Tables. Applied Mechanics and Materials, 2016, 826:6.

[10] Zou Y, Xu Y, Hu L, et al. Minimally invasive positioning robot system of femoral neck hollow screw implants based on x-ray error correction. Proceedings of the Spie, 2017, 245:102450D.

[11] Zahid S, Hobson P R, Cockerill D J A. A segmented anode vacuum phototriode with position sensitivity. Journal of Instrumentation, 2018, 13(01):C01014-C01014.

[12] Reiner B, Bownes P, Buckley D L , et al. Quantifying the trigger level of the vacuum surveillance system of the Gamma-Knife eXtend ${ }^{\mathrm{TM}}$ positioning system and evaluating the potential impact on dose delivery.. Journal of Radiosurgery \& Sbrt, 2016, 4(1):31-42.

[13] Lehman C D, Shook J E. Position of clip placement after vacuum-assisted breast biopsy: is a unilateral two-view postbiopsy mammogram necessary? Breast Journal, 2015, 9(4):272-276.

[14] Ayhan Üngördü, Tezer N. Effect on frontier molecular orbitals of substituents in 5-position of uracil base pairs in vacuum and water. Journal of Theoretical and Computational Chemistry, 2017, 16(07):7.

[15] Ashwal E, Wertheimer A, Aviram A, et al. The association between fetal head position prior to vacuum extraction and pregnancy outcome. Archives of Gynecology and Obstetrics, 2016, 293(3):567-573. 